Hautarzt $2021 \cdot 72: 533-534$

https://doi.org/10.1007/s00105-021-04841-1

Angenommen: 12. Mai 2021

(c) Springer Medizin Verlag GmbH, ein Teil von Springer Nature 2021

Wer wie Zugang zu einem geregelten Beruf erhält, insbesondere zu einem mit so großer Verantwortung für das Wohl anderer Menschen ausgestatteten Beruf wie dem des Arztes oder des Facharztes für Dermatologie, ist eine komplexe und am Ende hochpolitische Entscheidung.

Die in Artikel 12 des Grundgesetzes gewährleistete Freiheit der Berufswahl setzt - außer bei ungelernten Berufen eine Ausbildung als Vorstufe einer Berufsaufnahme voraus. „Beide sind integrierende Bestandteile eines zusammengehörenden Lebensvorganges“, wie das Bundesverfassungsgericht in seiner wegweisenden Entscheidung zum Numerus clausus in der Medizin 1972 ausführte (BVerfGE 33, 303). Die Regelungsbefugnis für den Zugang zum Arztberuf und damit zur Definition der Prüfungsanforderungen hat der Bundesgesetzgeber, der diese mit der Approbationsordnung umsetzt; die Erfüllung der Anforderungen wird in einem Staatsexamen geprüft. $\mathrm{Da}$ - anders als in der Vergangenheit - eine selbstständige ärztliche Tätigkeit auf der Basis des Medizinstudiums und des Staatsexamens allein nicht möglich ist, schließen sich vor Aufnahme einer selbstständigen Tätigkeit eine Weiterbildung und die Anerkennung als Facharzt an, die sich nach den Kammerbzw. Heilberufsgesetzen der Länder und den Weiterbildungsordnungen der Landesärztekammern richten. Die ärztliche Weiterbildung beinhaltet damit das Erlernen spezieller ärztlicher Fähigkeiten und Fertigkeiten auf den medizinischen Fachgebieten. In den Weiterbildungsordnungen werden dafür Mindestzeiten und Weiterbildungsinhalte vorgegeben, wobei diese in der neuen Musterweiterbil-

\title{
P. Elsner
}

Klinik für Hautkrankheiten, Universitätsklinikum Jena, Jena, Deutschland

\section{Die neue Weiterbildungsordnung Dermatologie: Neuer Wein in alten Schläuchen?}

dungsordnung von 2018 in „Kognitive und Methodenkompetenz" (Kenntnisse) und „Handlungskompetenz" (Erfahrungen und Fertigkeiten) unterschieden werden. Der lerntheoretische Hintergrund dieser Kompetenzorientierung wird im Beitrag von Falk Ochsendorf und Julia Welzel in diesem Heft in Der Hautarzt sehr schön dargestellt. Dass diese Unterscheidung von Kenntnissen und Fertigkeiten für den Arztberuf, der zwar auf wissenschaftlich-theoretischen Erkenntnissen beruht, diese aber für den konkreten Patienten anwenden muss, sinnvoll ist, braucht nicht eigens betont $\mathrm{zu}$ werden. Ein Arzt, der die Wirkung eines Medikamentes versteht, aber nicht in der Lage ist, dieses korrekt und sicher anzuwenden, kann eher Schaden als Nutzen anrichten.

Neu ist das alles freilich nicht, denn auch in der Vergangenheit erlernten Ärzte in Weiterbildung auf der Basis theoretischer Kenntnisse deren praktische Umsetzung. Frühere Versionen der Weiterbildungsordnung enthielten, wie heute auch, bestimmte Mindestanforderungen und -leistungen, die - unter Aufsicht erbracht werden mussten, wie etwa Operations- oder Diagnostikzahlen.

Mit der neuen „Kompetenzorientierung" entsteht also ein schöner lerntheoretischer Überbau. Viel interessanter allerdings ist, wie weit sich die Praxis ändert.

Eine Änderung der Praxis der ärztlichen Weiterbildung zum Guten würde zuallererst eine ehrliche Bestandsaufnahme voraussetzen. Wie sieht diese aus? Im Jahr 2014 berichtete der Marburger Bund über die Ergebnisse einer repräsentativen Umfrage zur Weiterbildungssituati- on bei Ärzten in Weiterbildung [1]. Danach würde weniger als die Hälfte der Ärzte (47\%) ihre Weiterbildungsstätte weiterempfehlen; $23 \%$ der Befragten gaben an, nicht von ,ihren Weiterbildungsermächtigten“, sondern überwiegend von anderen Ärzten in der Weiterbildung angeleitet zu werden. Die Weiterbildungsinhalte konnten laut $63 \%$ der Befragten im klinischen Alltag nur unzureichend erworben werden, und $85 \%$ der Assistenzärzte hatten keinen strukturierten Weiterbildungsplan. Nur 9\% der Assistenten berichteten über ein regelmäßiges Feedback. Immerhin fühlten sich $43 \%$ der Befragten von ihrem Weiterbilder gefördert, und $50 \%$ hielten ihren Weiterbilder für didaktisch kompetent.

Es ist nicht anzunehmen, dass sich an diesen Einschätzungen der Assistenten in der Zwischenzeit viel geändert hat. Dass viele Weiterbildungszeugnisse eher Wunschvorstellungen als tatsächlich vermittelte Inhalte bescheinigen, ist ein offenes Geheimnis, das das traurige Resümee bestätigt, dass mit der ärztlichen Weiterbildung in Deutschland offensichtlich etwas im Argen liegt.

Das Problem ist dabei nicht der ,theoretische Überbau" in Form der 2018 novellierten Weiterbildungsordnung, sondern, um mit Marx zu sprechen, die reale Basis der „Produktionsverhältnisse“, über denen sich dieser Überbau erhebt. Die reale Basis der ärztlichen Weiterbildung in Deutschland ist, dass diese nicht eigens finanziert wird - mit der Ausnahme von Weiterbildungsstellen vorwiegend im hausärztlichen Bereich, die durch kassenärztliche Vereinigungen gesichert werden. Realität ist, dass Assistenzärzte in Kliniken eingestellt werden, 
um medizinische Leistungen unter den Bedingungen der DRGs zu erbringen, und nicht zum Zwecke ihrer Weiterbildung. Sie werden weitergebildet von weiterbildungsermächtigten (Chef-)Ärzten, die ebenfalls nicht vorrangig zur Weiterbildung junger Ärzte beschäftigt werden, sondern zur Krankenversorgung, ökonomisch ausgedrückt: zur Erbringung von Fallzahlen oder Prozeduren. Eine Vergütung für die Weiterbildung von Ärzten erhalten die Kliniken eben gerade nicht.

Damit ist die ärztliche Weiterbildung in Deutschland am Ende Privatangelegenheit, nämlich der jungen Kollegen, die sich weiterbilden wollen, ohne dafür bezahlt zu werden, und der Weiterbildungsermächtigten, die dies in kollegialer Verbundenheit und aus persönlichem Engagement mehr oder weniger gut tun. Aus gutem Grund wird die Qualität der ärztlichen Weiterbildung daher nicht ernsthaft kontrolliert; wollte man dies tun, müsste man sich nämlich der Frage stellen, wie diese überhaupt finanziert ist.

Andere Länder gehen hier - seit Langem - andere Wege. So erfolgt etwa in den USA die Finanzierung der Weiterbildungsstellen im Wesentlichen durch das Gesundheitsprogramm Medicare („Direct Medical Education" [DME] als Zahlung der Gehälter der Weiterbildungsassistenten). Auch in den Niederlanden wird dieärztliche Weiterbildung vollständig von der Regierung finanziert. Krankenhäuser erhalten Mittel zur Deckung der Kosten für die Gehälter der Assistenzärzte und den Ausbildungsaufwand der Weiterbildungsermächtigten [2]. Die Anzahl der Assistenzarztstellen wird von der Regierung festgelegt, wie dies letztlich auch in den USA der Fall ist, wo Weiterbildungsstätten regelmäßig besucht und daraufhin evaluiert werden, welche Assistentenzahl sie hochqualitativ weiterbilden können. In seiner eigenen Zeit als Dermatologe in den USA hat der Autor erlebt, dass die Weiterbildungsassistenten in 1:1-Betreuung jeweils einem Facharzt zugeordnet waren, unter dessen Aufsicht sie tätig werden durften. Jeder Vormittag begann für die „Residents“ mit einer „Resident Lecture“, in der von Experten das dermatologische Fachwissen vermittelt und intensiv diskutiert wurde. Ein Halbtag pro Woche war den „Grand Rounds“ vorbehalten, bei denen nach einer Vorlesung eine Patientenvorstellung mit anschließender Diskussion in "großer Runde“ mit Chef, Professoren und Fachärzten erfolgte.

Von einer solchen - sicherlich fordernden - hochqualitativen Weiterbildung in der Dermatologie können wir in Deutschland in der Fläche nur träumen, auch wenn einzelne Kliniken am Ende einen ebenbürtigen Standard aufweisen mögen.

Der Erfolg einer ärztlichen Weiterbildung sollte auch nicht allein auf der Basis eines - wie gut auch immer geführten - „e-Logbuches“ oder einer Facharztprüfung in Form eines halbstündigen „kollegialen Gespräches“ evaluiert werden. Wünschenswert wäre ein ehrliches, transparentes, faires Examen. Die Dermatologie hat dafür mit dem „Europäischen Facharztexamen“ eine Vorlage geliefert [3], das von deutschen Ärzten allerdings nur sporadisch, weil nicht verpflichtend, angenommen wird. Ergänzt werden müsste dieses theoretische Examen durch eine praktische Kompetenzprüfung, etwa in Form einer OSCE-Prüfung.

Nehmen wir doch einmal die Luftfahrt als Parallele. Niemand möchte von Verkehrspiloten geflogen werden, die ihre Ausbildung „nebenbei“ absolviert haben und deren Prüfung sich auf ein freundliches Gespräch beschränkt hat. Wir wollen sicher fliegen und von Piloten transportiert werden, die ihre Kompetenz theoretisch und praktisch in allen Luftlagen, auch kritischen, demonstriert haben.

Warum sollen in der Medizin weniger strenge Maßstäbe gelten?

Dass nach der neuen Weiterbildungsordnung für die Dermatologie der Kompetenzerwerb auch rein ambulant möglich ist, erscheint da eher zweitrangig. Tatsächlich ist es für Assistenzärzte nicht in allen weiterbildungsberechtigten Praxen selbstverständlich, zumindest eine einzige seriöse dermatologische Fachzeitschrift zur Verfügung zu haben und das breite Leistungsspektrum der Dermatologie zu erfahren und zu erlernen, wie es in vielen Kliniken (noch, aber mit leider rückläufiger Tendenz) angeboten wird. Anstatt uns jedoch in Debatten um ambulante oder stationäre Weiterbildung zu verheddern, sollten wir uns als Dermatologen besser ehrlich machen und konstatieren, dass wir eine schonungslose Bestandsaufnahme der ärztlichen Weiterbildung und - darauf basierend - mutige Reformvorschläge benötigen, nicht nur für die Dermatologie, sondern für die gesamte Medizin in Deutschland, und damit letztlich zum Wohl der uns anvertrauten und sich uns anvertrauenden Patienten.

Ihr

\section{Peter Elsner}

\section{Korrespondenzadresse}

Prof. Dr. P. Elsner

Klinik für Hautkrankheiten, Universitätsklinikum Jena

Erfurter Str. 35, 07743 Jena, Deutschland

elsner@derma-jena.de

Interessenkonflikt. P. Elsner ist Direktor einer Universitäts-Hautklinik und Weiterbildungsermächtigter der Thüringer Landesärztekammer für Dermatologie.

\section{Literatur}

1. Deutscher Ärzteverlag Gmb HRDÄ (2014) Weiterbildung zum Facharzt: Der Nachwuchs ist unzufrieden. https://www.aerzteblatt.de/archiv/159928/ Weiterbildung-zum-Facharzt-Der-Nachwuchsist-unzufrieden. Zugegriffen: 12. Febr. 2018

2. Hoff RG, Frenkel J, Imhof SM, Ten Cate O (2018) Flexibility in postgraduate medical training in the Netherlands. Acad Med 93:S32-S36

3. UEMS European Board of Dermatovenereology Diploma Examination (2021) https://www. uems-ebdv.org/web/index.php/uems-boardexamination.Zugegriffen: 11. Mai 2021 